\title{
Analysis of CARD10 and CARD11 somatic mutations in patients with ovarian endometriosis
}

\author{
YANG ZOU $^{1,2}$, JIANG-YAN ZHOU $^{1,3}$, FENG WANG $^{1,2}$, ZI-YU ZHANG $^{1,2}$, FA-YING LIU $^{1,2}$, \\ YONG LUO ${ }^{1,2}$, JUN TAN ${ }^{1,4}$, XIN ZENG $^{1}$, XI-DI WAN ${ }^{1}$ and OU-PING HUANG ${ }^{1,3}$ \\ ${ }^{1}$ Key Laboratory of Women's Reproductive Health of Jiangxi Province; ${ }^{2}$ Central Laboratory; ${ }^{3}$ Department of Gynecology; \\ ${ }^{4}$ Reproductive Medicine Center, Jiangxi Provincial Maternal and Child Health Hospital, Nanchang, Jiangxi 330006, P.R. China
}

Received October 11, 2017; Accepted March 27, 2018

DOI: $10.3892 / \mathrm{ol} .2018 .8659$

\begin{abstract}
Endometriosis is a complex and heterogeneous pre-malignant inflammatory disease harboring multiple gene mutations. Previous studies have suggested that caspase recruitment domain family member (CARD)10 and CARD11 mutations may exist in endometriosis. In the present study, a collection of endometriotic lesions and paired peripheral blood from 101 patients with ovarian endometriosis were obtained, and the entire coding sequences of the CARD10 and CARD11 genes were sequenced. Evolutionary conservation analysis and online prediction programs were applied to analyze the disease-causing potential of the identified mutations. A total of 4 novel somatic mutations were identified in 4 out of the $101(4.0 \%)$ samples: 2 in-frame deletions in CARD10 (c.785_790delAGGAGA, p.K272 E273delKE; c.785_802delAGGAGAAGGAGAAGGAGA, p.K272_V277delKEPDNV) and 2 heterozygous missense mutations in CARD11 (c.49G>T, p.D17Y; c.160G $>$ C, p.E54Q). The sample with CARD10 p.K272_E273delKE deletion was obtained from a 47 -year-old patient who was also diagnosed with uterine leiomyoma, while the CARD10 p.K272_V277delKEPDNV-mutated sample was from a 43-year-old patient exhibiting a decreased blood eosinophil granulocyte ratio $(0.3 \%)$ and an elevated serum creatine kinase level (314 U/l). The patient with the CARD11 p.D17Y mutation was 38 years old and exhibited an increased level of cancer antigen $125(45.4 \mathrm{U} / \mathrm{ml})$, while the patient with the CARD11 p.E54Q mutation was 46 years old and exhibited no other gynecological conditions. Evolutionary conservation analysis and online prediction programs suggested that these mutations may be disease-causing. In summary, 4 novel somatic mutations in the CARD10 and CARD11 genes were identified from
\end{abstract}

Correspondence to: Professor Ou-Ping Huang, Key Laboratory of Women's Reproductive Health of Jiangxi Province, Jiangxi Provincial Maternal and Child Health Hospital, 318 Bayi Avenue, Nanchang, Jiangxi 330006, P.R. China

E-mail: huangouping@gmail.com

Key words: CARD10, CARD11, mutations, ovarian endometriosis amongst 101 cases of ovarian endometriosis for the first time, these mutations may serve active roles in the development of ovarian endometriosis.

\section{Introduction}

Endometriosis is a heterogeneous estrogen-dependent chronic gynecological disease in women of reproductive age (1-3). The condition is characterized by endometrial tissues ectopically implanting outside the uterine cavity, and is subdivided mainly into ovarian, peritoneal and deep infiltrating endometriosis according to the different implant locations $(4,5)$. The most common clinical symptoms of endometriosis include dysmenorrhea, chronic pelvic pain and diminished fertility potential, which greatly influence the quality of life of affected individuals (6). Despite numerous studies being performed on this research field, the molecular etiology of endometriosis is not yet fully understood $(7,8)$.

It has long been accepted that endometriosis is actually an inflammatory disease $(9,10)$, with cytokine levels that are elevated in the peritoneal fluid, serum and endometriotic lesion tissues (11-13). Based on these observations, certain researchers formulated the inflammation hypothesis that leukocytes are recruited by the endometrial stromal cells within endometriotic lesions, and that proinflammatory cytokines are then secreted from these leukocytes, which will facilitate the progression of endometriosis $(14,15)$. Furthermore, it has also been proposed that the changed progesterone responsiveness may be the main reason for the elevated production of cytokines in endometriosis (16-18). However, in spite of advances $(1,19)$, there have not been any therapeutic strategies targeting inflammatory factors in endometriosis that have been successfully applied to samples with endometriosis, implying a great underappreciation of the role of inflammation in endometriosis $(20,21)$.

Caspase recruitment domain family member 11 (CARD11) belongs to the CARD protein family; it can bind with B-cell CLL/lymphoma 10 and activate the inflammation-associated nuclear factor $\kappa \mathrm{B}$ (NF- $\kappa \mathrm{B}$ ) signaling pathway (22). Furthermore, CARD11 also acts as a scaffold protein to assist the assembly of multiprotein signaling molecules in the plasma membrane (23). Previous studies showed that CARD11 somatic mutations were detected frequently in multiple human cancer types, including hematological malignancies, colorectal cancer and malignant 
melanoma; the mutations were distributed along the CARD11 coding sequence while being mainly clustered within the coiled-coil domain (24-28). Most recently, a somatic mutation in the CARD11 gene (p.R30W, c.C88T) was identified in the endometriotic lesion of 1 out of 16 samples with ovarian endometriosis. Considering the fact that endometriosis is a potential premalignant disorder and the endometriotic lesions harbored certain genetic alterations in tumor-associated genes (29-33), and furthermore that mutations in the paralogous genes usually occurred in the same cancer types $(34,35)$, we thus hypothesized that mutations in the paralogous CARD10 and CARD11 may exist in ovarian endometriosis.

In the present study, a total of 101 patients with ovarian endometriosis were recruited and analyzed for the presence of CARD10 and CARD11 mutations, with the aim of exploring the potential involvement of mutations in these two genes in the pathogenesis of ovarian endometriosis.

\section{Materials and methods}

Samples. The endometriotic lesions of the ovaries and paired peripheral blood samples were obtained immediately following surgical resection from a total of 142 patients with ovarian endometriosis who were treated in the Department of Gynecology, Jiangxi Provincial Maternal and Child Health Hospital (Nanchang, Jiangxi, China), between July 2015 and January 2018. All samples were diagnosed pathologically by two experienced pathologists, and the 101 cases with an ectopic endometrium purity of $>30 \%$ were included in the present study. The study was approved by the Institutional Review Board of Jiangxi Provincial Maternal and Child Health Hospital, and the detailed protocol was conducted according to the Declaration of Helsinki and the Jiangxi Provincial Maternal and Child Health Hospital. Written informed consent was obtained from all patients prior to the study.

Mutational analysis of the CARD10 and CARD11 genes. The genomic DNA was isolated from the endometriotic lesions and paired peripheral blood samples using the DNeasy Blood and Tissue kit (catalog no. 69504; Qiagen, Inc., Valencia, CA, USA) according to the manufacturer's protocols. The entire coding region of the CARD10 and CARD11 genes in the endometriotic lesions of patients with ovarian endometriosis were amplified by polymerase chain reaction (PCR) with sets of primer pairs (Table I). In brief, for each PCR amplification reaction, $\sim 50 \mathrm{ng}$ total DNA was used in a final volume of $30 \mu \mathrm{l}$, with the following amplification protocols: An initial pre-denaturation step at $94^{\circ} \mathrm{C}$ for $3 \mathrm{~min}$, followed by 35 cycles of denaturation at $94^{\circ} \mathrm{C}$ for $30 \mathrm{sec}$, annealing at different temperatures $\left(52-60^{\circ} \mathrm{C}\right.$; Table I) for $30 \mathrm{sec}$ and extension at $72^{\circ} \mathrm{C}$ for $30 \mathrm{sec}$, with a final extension at $72^{\circ} \mathrm{C}$ for $7 \mathrm{~min}$. PCR was performed in a Thermal Cycler 2720 (Applied Biosystems; Thermo Fisher Scientific, Inc., Waltham, MA, USA). The PCR amplification products were then purified and sequenced on an ABI Prism 3730 DNA sequencer (Applied Biosystems; Thermo Fisher Scientific, Inc.). An additional independent PCR amplification and DNA sequencing was performed in the endometriotic lesions for samples harboring potential CARD10 or CARD11 mutations. The statuses of the potential somatic mutations were verified by sequencing the DNA sequences of the paired peripheral blood in the same patients. The procedure of PCR amplification and DNA sequencing was performed as described above.

Evolutionary conservation analysis. The protein sequences from 21 vertebrate species from GenBank (https://www.ncbi. nlm.nih.gov/genbank/) were used to analyze the evolutionary conservation status of the identified CARD11 mutations, including Homo sapiens (NP_115791), Macaca mulatta (XP_014988691), Rattus norvegicus (XP_017454079), Mus musculus (NP_780571), Dipodomys ordii (XP_012878958), Bos taurus (NP_001103266), Microcebus murinus (XP_012614839), Equus asinus (XP_014702187), Pan paniscus (XP_008971413), Cavia porcellus (XP_013008240), Camelus ferus (XP_014412591), Ovis aries (XP_014959439), Mustela putorius furo (XP_0129032), Canis lupus familiaris (XP_005621), Gallus gallus (NP_001006161), Calidris pugnax (XP_014801661), Ficedula albicollis (XP_005054487), Lipotes vexillifer (XP_007467483), Apteryx australis mantelli (XP_013797455), Stegastes partitus (XP_008283939) and Ictalurus punctatus (XP_017310081). Multiple sequence alignment was performed using the 'ClustalW' tool of the alignment function in the Molecular Evolutionary Genetics Analysis software (MEGA, version 4.0) which was created and developed by Kumar et al (36).

Bioinformatics programs prediction of the CARDIO and CARD11 mutations. PolyPhen-2 (http://genetics.bwh.harvard. edu/pph2/) (37) and MutationTaster (http://mutationtaster. org/) (38) software were used to analyze the disease-causing potential for the identified missense mutations, while the SIFT (http://sift.jcvi.org/) program (39) was used to predict the potential pathogenicity of the in-frame deletions. All bioinformatic analysis was performed on February 6th, 2018. Together, the software automatically assessed each mutation as either pathogenic or benign.

Statistical analysis. The frequency difference of CARD11 mutation in the current study and the previous study (29) was analyzed by two-tailed Fisher's exact test using SPSS software version 18.0 (SPSS Inc., Chicago, IL, USA). P<0.05 was considered to indicate a statistically significant difference.

\section{Results}

CARD10 and CARDI1 mutations in ovarian endometriosis. The median age of the sample cohort was 43 years (range, 21-56 years). A total of 4 somatic mutations in CARD10 or CARD11 genes were identified in 4 out of 101 endometriotic lesions from the patients with ovarian endometriosis, with the somatic status confirmed compared with that of the paired peripheral blood (Fig. 1). The 2 mutations in CARD10 (c.785_790delAGGAGA, p.K272_E273delKE; c.785_802delAGGAGAAGGAGAAGGAGA, p.K272_ V277delKEPDNV) caused in-frame deletions, while the 2 mutations in CARD11 (c.49G>T, p.D17Y; c.160G >C, p.E54Q) were missense and heterozygous. It should be noted that a previous study identified a CARD11 somatic mutation (p.R30W) in 1 out of $16(6.3 \%)$ patients with ovarian endometriosis, but no CARD10 mutations (29). In the present study, the sample with the CARD10 p.K272_E273delKE deletion 
Table I. Polymerase chain reaction primers for the amplification of the CARD10 and CARD11 genes.

\begin{tabular}{|c|c|c|c|c|}
\hline Gene and exon & Forward primer $\left(5^{\prime}-3^{\prime}\right)$ & Reverse primer $\left(5^{\prime}-3^{\prime}\right)$ & Annealing, ${ }^{\circ} \mathrm{C}$ & Amplicon, bp \\
\hline \multicolumn{5}{|l|}{ CARD10 } \\
\hline 1 & ACATCTAGCCCTAGGGAGCC & СССАСТCTACTGATGCGGAG & 52 & 433 \\
\hline 2 & CCCCTAGACCCTGGGTACAT & CTTCAGCCTCTCCATGCCTC & 52 & 288 \\
\hline 3 & GAGCTGCCTATTCTGTCCCC & CGTCAAAGAGCCAGCAATGG & 52 & 468 \\
\hline 4 & GCTTCGGCTTCTCTGGGAAT & CCACAAGCCCCAGTACCTG & 58 & 405 \\
\hline $5-6$ & CTGGTCAGGTGGGTTTGGAG & GTCCTAACCCAACAGGGAGC & 52 & 1,088 \\
\hline 7 & TAATTTAGCTCAGGGCCCGG & CTCCACAATCTTGACCCGCT & 56 & 358 \\
\hline $8-9$ & CACATGGCAGGTGCTCAGTA & GCTATCCCCAGCCATCTCAC & 52 & 687 \\
\hline $10-11$ & CTTGGGGGCTGTGAGACATT & TAAAACAGGGGCGAGGCAAT & 60 & 815 \\
\hline $12-13$ & AGGCCTGGGGGTGGGAGTCA & CAGGAGAAGGGCAATTGG & 55 & 976 \\
\hline $14-15$ & GAAGGTCGGGTCCTGGGAA & TGGGCACATAGTAGATGACCA & 52 & 756 \\
\hline 16 & AGGCTCTTCTGGCAAGCCT & AGCATGACCCCCACTCCTGT & 56 & 372 \\
\hline $17-19$ & TGGGGTATCGACGAGCTG & AGAGAGGCATCCTCAAGGA & 60 & 1,189 \\
\hline 20 & АТСТTСТСТСТСТGАСТСТ & TGAGGAGAGTGATGGGGAC & 58 & 1,192 \\
\hline \multicolumn{5}{|l|}{ CARD11 } \\
\hline 3 & GCTGTTCCAGTGAGACTGCT & GACTGCGGACCCCAGTTTAA & 54 & 394 \\
\hline 4 & TGAGACCAGCCACAGAGACT & GTGGTTGACAGACCCCAGTT & 56 & 366 \\
\hline 5 & CTGCGTCTGGAACCTCCTTT & СCTGCACCTGCTTTATGGGA & 56 & 482 \\
\hline 6 & GGGAAGCGTTGCCTTTTCTG & CAGGTTCATCGTTTCCCCCA & 54 & 326 \\
\hline $7-8$ & CCAGGCACAAAACCTTCTGC & AAACACTCTGAAGGAGCCGG & 60 & 1,132 \\
\hline 9 & GATGATGCCTGTCCCTGGG & CTTCAGGCGTGGGGTCCT & 52 & 314 \\
\hline 10 & CCATCAGCCCAGCCATCTT & CCAAGACCCACCCAGAAGC & 52 & 371 \\
\hline 11 & AAGCCCCAGTGACATGTGTC & CGCAGGATTGTTCGTTACAG & 52 & 226 \\
\hline 12 & СТССССТСТСТСТСТТССА & GCTGGGTCCCTGGATGGCA & 60 & 324 \\
\hline 13 & TGCCGCCTGAGTAGGAGGC & GAGGACAGCTGGGTCAGCA & 55 & 305 \\
\hline 14 & ССТCTCAGAAGCAAGGCCAC & AGGCTTATCTTTTGTGTTC & 57 & 312 \\
\hline 15 & GACCAGCCCAGCAGGTCCC & CCAGGAAGTGATTTCTGACT & 55 & 369 \\
\hline $16-17$ & CACCCAGGCGCCTGATGAC & GCCTTCCACTGAACAGACGA & 52 & 950 \\
\hline $18-19$ & CTAAGAGCAGCATATTGCACA & GAATTCATCATCCCAATACGG & 52 & 1,363 \\
\hline 20 & CTAAGAAAGCGTTAGCAT & CACTGTGAAGAGTTGCAAGT & 58 & 351 \\
\hline 21 & TGCCGGGTTGAGGGCAGCA & GACCGATGTTCCTCCAGGT & 52 & 299 \\
\hline $22-23$ & ACACTGACGGTGGCTTCCA & TTGTCCCTGGCCCAGGTG & 58 & 1,477 \\
\hline 24 & CCTGCATCCAGGCCCCGCT & GGTCAGTCCCGTACTTGGTG & 54 & 336 \\
\hline 25 & TGGTGCCACACCAGCGCCA & AGCGTCTGCTGGGGCAGCTC & 52 & 391 \\
\hline
\end{tabular}

CARD, caspase recruitment domain family member.

was from a 47-year-old woman who was also diagnosed with uterine leiomyoma, while the sample with the CARD10 p.K272_V277delKEPDNV mutation was from a 43-year-old patient exhibiting a decreased blood eosinophil granulocyte ratio $(0.3 \%$ ) (normal range: $0.4-8 \%$ ) and elevated serum creatine kinase level (314 U/l) (normal range for women: 96-140 U/1). The patient with the CARD11 p.D17Y mutation was 38 years old and exhibited an increased level of cancer antigen 125 (45.4 U/ml) (normal range: 0-35 U/ml), while the patient with the CARD11 p.E54Q mutation was 46 years old and exhibited no other apparent gynecological conditions.

Evolutionary conservation analysis of CARD11 missense mutations. The evolutionary conservation analysis of CARD11 in 21 vertebrate species ranging from Homo sapiens to Ictalurus punctatus showed that the two mutated amino acid residues (p.D17, p.E54) were highly conserved (Fig. 2).

Causative potential of CARD10 and CARD11 mutations. The PolyPhen-2 and MutationTaster online prediction programs were used to predict the disease-causing potentials of the detected missense mutations. The CARD11 p.D17Y (c.49G $>$ T) and p.E54Q (c.160G $>$ C) mutations were predicted to be possibly damaging with a score of $>0.77$ by Polyphen- 2 software, while predicted to be disease-causing with a score of $>29$ and a probability of $>0.99999$ by MutationTaster. For the two in-frame deletions in CARD10, p.K272 V277delKEPDNV was predicted to be disease-causing, while 

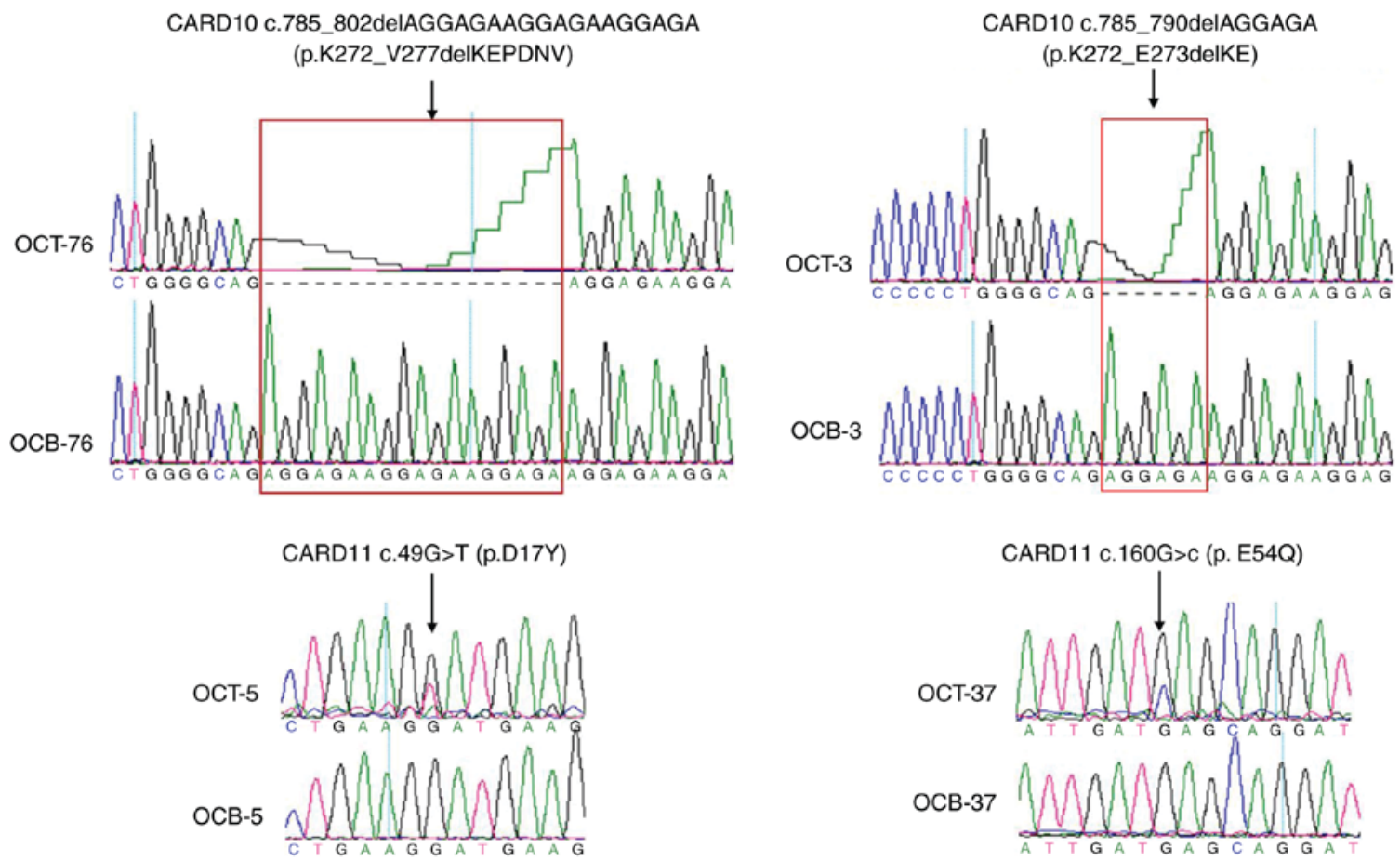

Figure 1. Sequencing electropherograms of CARD10 and CARD11 mutations. The arrows indicate the locations of the identified mutations. OCT, ovarian endometriotic lesions; OCB, paired peripheral blood from ovarian endometriosis samples; CARD, caspase recruitment domain family member.

Homo sapiens
Mus musculus
Rattus norvegicus
Gallus gallus
Bos taurus
Macaca mulatta
Mustela putorius furo
Calidris pugnax
Pan paniscus
Cavia porcellus
Ovis aries
Dipodomys ordii
Canis lupus familiaris
Ficedula albicolis
Camelus ferus
Microcebus murinus
Lipotes vexillifer
Apteryx australis mantelli
Equus asinus
Stegastes partitus
Ictalurus punctatus

CARD11 p.D17

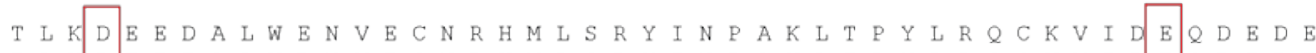
T L K D E E E A L W D N V E C N R H M L S R Y I N P A K L T P Y L R Q C K V I D E

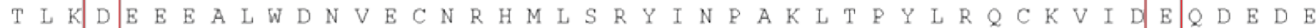
T L K D E E E E A L W E N V E C N R H M L S R Y I N P A K L T P Y L R $Q$ C C K V I I D E

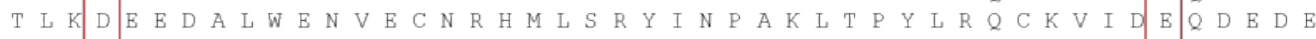

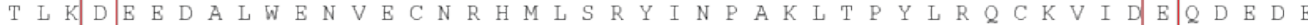

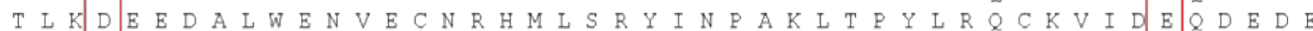

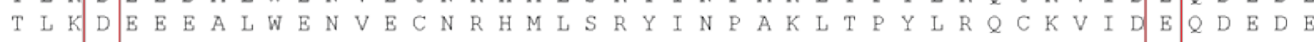
T L K D E E D D A L W E N V E C N R H M L S R Y I N P A K L T P Y L R Q C K V I D E

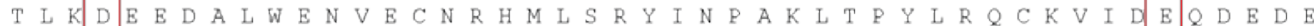
T L K D E E D A L W E N V E C N R H M L S R Y I N P A K L T P Y L R Q C K V I D E

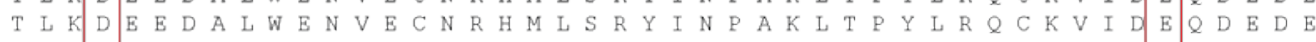

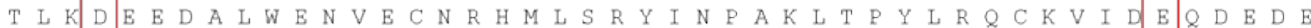

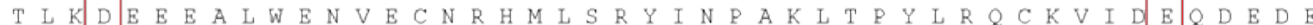

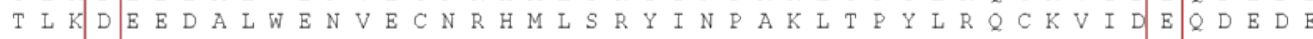

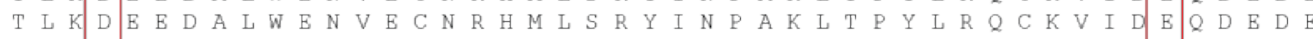

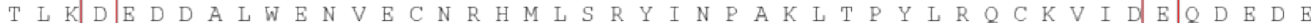

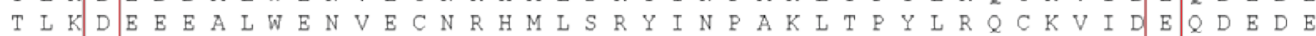

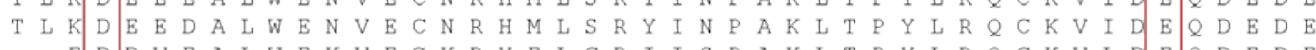
- F D D V E A L W E K V E G K R Y E L C R I I S P A K L T P Y L R Q C K V L D E $N$ I L D D E E A L W E N V E K N R Y I L C R Y I N P N K L T S Y L R $Q$ C C K V I I D E E

Figure 2. Evolutionary conservation analyses of the CARD11 missense mutations. The CARD11 protein sequences of 21 vertebrate species from the GenBank database were adopted to perform the evolutionary conservation analysis. The p.D17Y and p.E54Q mutations in CARD11 were highly conserved among these vertebrates. CARD, caspase recruitment domain family member.

p.K272_E273delKE may be benign, according to the results predicted by the SIFT program. None of the mutations were found in the dbSNP (https://www.ncbi.nlm.nih.gov/snp), ExAC (http://exac.broadinstitute.org/) or 1000 Genomes Project (http://www.internationalgenome.org/) databases.

\section{Discussion}

Previous studies revealed that CARD10 and CARD11 served as scaffold proteins to regulate the activation of the $\mathrm{NF}-\kappa \mathrm{B}$ signaling pathway under different cellular contexts, by recruiting and activating the $N F-\kappa B$ repressor $I \kappa B$ kinase $(22,40)$. When considering that i) endometriosis is an inflammation-related premalignant disease harboring somatic mutations in multiple genes $(9,10)$, ii) CARD11 somatic mutations exist in ovarian endometriosis (29) and iii) mutations in the paralogous genes occur frequently in multiple human malignancies $(34,35)$, the present study thus attempted to investigate the potential mutational spectra of the paralogous CARD10 and CARD11 genes in patients with ovarian endometriosis.

Frequent CARD11 mutations were detected first in diffuse large B cell lymphoma (41) and then also identified in melanoma, colorectal and endometrial cancer (26-28). Besides 
being found in these different cancer types, a previous study also identified a CARD11 somatic mutation (p.R30W) in 1 out of $16(6.3 \%)$ patients with ovarian endometriosis (29). In the present study, 2 novel CARD11 somatic mutations (p.D17Y and p.E54Q) were detected in 2 out of 101 (2.0\%) ovarian endometriosis cases; the observed frequency in the current study did not reach a statistically significant level when compared with that in the prior study $(\mathrm{P}=0.40)(29)$. All the identified mutations in the present study were somatic and not found in the dbSNP, 1000 Genome Project or ExAC databases. The evolutionary conservation analysis showed that the mutated amino acids in CARD11 (p.D17Y and p.E54Q) were highly conserved in 21 vertebrate species. Furthermore, these mutations were predicted to be disease-causing by Polyphen-2 and MutationTaster. It should be mentioned that a somatic mutation in the 54th codon of CARD11, p.E54V (c.161A>T), was previously identified in a patient with hepatocellular carcinoma (42). Taken together, these results indicated that these somatic mutations in CARD11 may serve active roles in the development of ovarian endometriosis.

Besides CARD11 mutations, 2 somatic in-frame deletions in CARD10 were also identified in 2 out of 101 (2.0\%) cases of ovarian endometriosis. Since the indel mutations could be predicted by SIFT, but not by Polyphen-2 and MutationTaster, the p.K272_V277delKEPDNV deletion was predicted to be disease-causing, while the p.K272_E273delKE deletion was predicted to be benign by SIFT only. By contrast, Li et al (29) did not identify any CARD10 mutations in 16 cases with ovarian endometriosis, and we speculate that the relatively small sample size may be a main reason for this discrepancy. To the best of our knowledge, the present study is the first to reveal that CARD10 somatic mutations also exist in ovarian endometriosis. It should be noted that the p.K272_E273delKE somatic deletion has also previously been identified in a cancerous tissue from renal cell carcinoma (43) (http://cancer. sanger.ac.uk/cosmic). These results indicated that CARD10 p.K272_V277delKEPDNV deletion may facilitate the progression of ovarian endometriosis, while the role of the p.K272_E273delKE deletion requires further elucidation.

In the present study, the 4 somatic mutations in CARD10 and CARD11 were identified in 4 different individuals; this is consistent with previous observations that mutations in the paralogous genes are usually exhibited in a mutually exclusive manner $(34,35)$. The present study had several limitations. Firstly, the sample size was small, which may affect the mutation frequencies of the CARD10 and CARD11 genes, and the identified mutations should be verified in a larger sample size. Secondly, the study failed to obtain the clinical data showing whether samples with CARD10 or CARD11 mutations will display higher inflammation levels (such as interleukins) within the local endometriotic lesions or serum. Finally, further investigation is required to determine whether mutations would promote ovarian endometriosis via enhanced inflammation.

In summary, the present study identified 4 novel somatic mutations in CARD10 and CARD11 in 4.0\% (4/101) of patients with ovarian endometriosis for the first time. The results indicated that these mutations existed in a mutually exclusive manner and may serve a positive role in the pathogenesis of ovarian endometriosis.

\section{Acknowledgements}

Not applicable.

\section{Funding}

This study was supported by grants from the Natural Science Foundation of China (no. 81560255) and Jiangxi Province (nos. 20161ACB21021, 20151BAB205011 and 20143ACG70016).

\section{Availability of data and materials}

All data generated or analyzed during this study are included in this published article.

\section{Authors' contributions}

YZ performed mutation detection and manuscript preparation. JYZ and FW performed experiments. ZYZ performed conservation analysis. FYL and JT performed data analysis. YL performed mutation analysis. XZ and XDW performed sample collection. OPH performed study design and manuscript revision.

\section{Ethics approval and consent to participate}

The study was approved by the Institutional Review Board of Jiangxi Provincial Maternal and Child Health Hospital, and the detailed protocol was conducted according to the Declaration of Helsinki and the Jiangxi Provincial Maternal and Child Health Hospital. Written informed consent was obtained from all patients prior to the study.

\section{Consent for publication}

All the sample donors gave permission for the publication of their data prior to the study.

\section{Competing interests}

The authors declare that they have no competing interests.

\section{References}

1. Han SJ, Jung SY, Wu SP, Hawkins SM, Park MJ, Kyo S, Qin J, Lydon JP, Tsai SY, Tsai MJ, et al: Estrogen receptor $\beta$ modulates apoptosis complexes and the inflammasome to drive the pathogenesis of endometriosis. Cell 163: 960-974, 2015.

2. Ahn SH, Edwards AK, Singh SS, Young SL, Lessey BA and Tayade C: IL-17A contributes to the pathogenesis of endometriosis by triggering proinflammatory cytokines and angiogenic growth factors. J Immunol 195: 2591-2600, 2015

3. Kocbek V, Grandi G, Blank F, Wotzkow C, Bersinger NA Mueller MD, Kyo S and McKinnon BD: TNFa-induced IKK $\beta$ complex activation influences epithelial, but not stromal cell survival in endometriosis. Mol Hum Reprod 22: 768-777, 2016.

4. Chan RW, Ng EH and Yeung WS: Identification of cells with colony-forming activity, self-renewal capacity, and multipotency in ovarian endometriosis. Am J Pathol 178: 2832-2844, 2011.

5. Vouk K, Hevir N, Ribić-Pucelj M, Haarpaintner G, Scherb H, Osredkar J, Möller G, Prehn C, Rižner TL and Adamski J: Discovery of phosphatidylcholines and sphingomyelins as biomarkers for ovarian endometriosis. Hum Reprod 27: 2955-2965, 2012. 
6. Barbieri M, Somigliana E, Oneda S, Ossola MW, Acaia B and Fedele L: Decidualized ovarian endometriosis in pregnancy: A challenging diagnostic entity. Hum Reprod 24: 1818-1824, 2009.

7. Kim TH, Yoo JY, Wang Z, Lydon JP, Khatri S, Hawkins SM, Leach RE, Fazleabas AT, Young SL, Lessey BA, et al: ARID1A is essential for endometrial function during early pregnancy. PLoS Genet 11: e1005537, 2015.

8. Borrelli GM, Abrão MS, Taube ET, Darb-Esfahani S, Köhler C, Chiantera V and Mechsner S: (Partial) Loss of BAF250a (ARID1A) in rectovaginal deep-infiltrating endometriosis, endometriomas and involved pelvic sentinel lymph nodes. Mol Hum Reprod 22: 329-337, 2016.

9. Raible MD: Pathophysiology and treatment of endometriosis. Am J Hosp Pharm 38: 1696-1701, 1981.

10. Gazvani R and Templeton A: Peritoneal environment, cytokines and angiogenesis in the pathophysiology of endometriosis. Reproduction 123: 217-226, 2002.

11. Kang YJ, Jeung IC, Park A, Park YJ, Jung H, Kim TD, Lee HG, Choi I and Yoon SR: An increased level of IL-6 suppresses NK cell activity in peritoneal fluid of patients with endometriosis via regulation of SHP-2 expression. Hum Reprod 29: 2176-2189, 2014.

12. Suen JL, Chang Y, Chiu PR, Hsieh TH, His E, Chen YC, Chen YF and Tsai EM: Serum level of IL-10 is increased in patients with endometriosis, and IL-10 promotes the growth of lesions in a murine model. Am J Pathol 184: 464-471, 2014.

13. Jiang JF, Deng Y, Xue W, Zheng TP and Sun AJ: Increased expression of Interleukin 37 in the eutopic and ectopic endometrium of patients with ovarian endometriosis. Reprod Sci 23 244-248, 2016

14. Taylor RN, Kane MA and Sidell N: Pathogenesis of endometriosis: Roles of retinoids and inflammatory pathways. Semin Reprod Med 33: 246-256, 2015.

15. Taylor RN, Yu J, Torres PB, Schickedanz AC, Park JK, Mueller MD and Sidell N: Mechanistic and therapeutic implications of angiogenesis in endometriosis. Reprod Sci 16: 140-146, 2009.

16. Attia GR, Zeitoun K, Edwards D, Johns A, Carr BR and Bulun SE: Progesterone receptor isoform A but not $\mathrm{B}$ is expressed in endometriosis. J Clin Endocrinol Metab 85: 2897-2902, 2000.

17. Osteen KG, Bruner-Tran KL and Eisenberg E: Reduced progesterone action during endometrial maturation: A potential risk factor for the development of endometriosis. Fertil Steril 83: 529-537, 2005

18. Luk J, Seval Y, Kayisli UA, Ulukus M, Ulukus CE and Arici A Regulation of interleukin-8 expression in human endometria endothelial cells: A potential mechanism for the pathogenesis of endometriosis. J Clin Endocrinol Metab 90: 1805-1811, 2005

19. Nothnick W and Alali Z: Recent advances in the understanding of endometriosis: The role of inflammatory mediators in disease pathogenesis and treatment. F1000Res 5. pii: F1000 Faculty Rev-186, 2016.

20. Platteeuw L and D'Hooghe T: Novel agents for the medical treatment of endometriosis. Curr Opin Obstet Gynecol 26: 243-252, 2014.

21. Bruner-Tran KL, Herington JL, Duleba AJ, Taylor HS and Osteen KG: Medical management of endometriosis: Emerging evidence linking inflammation to disease pathophysiology. Minerva Ginecol 65: 199-213, 2013.

22. Yang YK, Yang C, Chan W, Wang Z, Deibel KE and Pomerantz JL: Molecular determinants of scaffold-induced linear ubiquitinylation of B cell lymphoma/leukemia 10 (Bcl10) during T cell receptor and oncogenic caspase recruitment domain-containing protein 11 (CARD11) signaling. J Biol Chem 291: 25921-25936, 2016.

23. Thome M and Tschopp J: TCR-induced NF-kappaB activation: A crucial role for Carma1, Bcl10 and MALT1. Trends Immunol 24 419-424, 2003

24. Davis RE, Ngo VN, Lenz G, Tolar P, Young RM, Romesser PB, Kohlhammer H, Lamy L, Zhao H, Yang Y, et al: Chronic active $\mathrm{B}$-cell-receptor signalling in diffuse large B-cell lymphoma. Nature 463: 88-92, 2010

25. da Silva Almeida AC, Abate F, Khiabanian H, Martinez-Escala E, Guitart J, Tensen CP, Vermeer MH, Rabadan R, Ferrando A and Palomero T: The mutational landscape of cutaneous $\mathrm{T}$ cell lymphoma and Sézary syndrome. Nat Genet 47: 1465-1470, 2015
26. Giannakis M, Hodis E, Jasmine $\mathrm{Mu} X$, Yamauchi $\mathrm{M}$, Rosenbluh J, Cibulskis K, Saksena G, Lawrence MS, Qian ZR, Nishihara R, et al: RNF43 is frequently mutated in colorectal and endometrial cancers. Nat Genet 46: 1264-1266, 2014.

27. Mouradov D, Sloggett C, Jorissen RN, Love CG, Li S, Burgess AW, Arango D, Strausberg RL, Buchanan D, Wormald S, et al: Colorectal cancer cell lines are representative models of the main molecular subtypes of primary cancer. Cancer Res 74: 3238-3247, 2014

28. Shain AH, Garrido M, Botton T, Talevich E, Yeh I, Sanborn JZ, Chung J, Wang NJ, Kakavand H, Mann GJ, et al: Exome sequencing of desmoplastic melanoma identifies recurrent NFKBIE promoter mutations and diverse activating mutations in the MAPK pathway. Nat Genet 47: 1194-1199, 2015.

29. Li X, Zhang Y, Zhao L, Wang L, Wu Z, Mei Q, Nie J, Li X, Li Y, Fu X, et al: Whole-exome sequencing of endometriosis identifies frequent alterations in genes involved in cell adhesion and chromatin-remodeling complexes. Hum Mol Genet 23: 6008-6021, 2014

30. Lee AW, Templeman C, Stram DA, Beesley J, Tyrer J, Berchuck A, Pharoah PP, Chenevix-Trench G and Pearce CL; Ovarian Cancer Association Consortium: Evidence of a genetic link between endometriosis and ovarian cancer. Fertil Steril 105 35-43, 2016.

31. Matsumoto T, Yamazaki M, Takahashi H, Kajita S, Suzuki E, Tsuruta $\mathrm{T}$ and Saegusa M: Distinct $\beta$-catenin and PIK3CA mutation profiles in endometriosis-associated ovarian endometrioid and clear cell carcinomas. Am J Clin Pathol 144: 452-463, 2015.

32. Worley MJ Jr, Liu S, Hua Y, Kwok JS, Samuel A, Hou L, Shoni M, Lu S, Sandberg EM, Keryan A, et al: Molecular changes in endometriosis-associated ovarian clear cell carcinoma. Eur J Cancer 51: 1831-1842, 2015.

33. Anglesio MS, Papadopoulos N, Ayhan A, Nazeran TM, Noë M, Horlings HM, Lum A, Jones S, Senz J, Seckin T, et al: Cancer-associated mutations in endometriosis without cancer. N Engl J Med 376: 1835-1848, 2017.

34. Yan H, Parsons DW, Jin G, McLendon R, Rasheed BA, Yuan W, Kos I, Batinic-Haberle I, Jones S, Riggins GJ, et al: IDH1 and IDH2 mutations in gliomas. N Engl J Med 360: 765-773, 2009.

35. Zou Y, Zeng Y, Zhang DF, Zou SH, Cheng YF and Yao YG: IDH1 and IDH2 mutations are frequent in Chinese patients with acute myeloid leukemia but rare in other types of hematological disorders. Biochem Biophys Res Commun 402: 378-383, 2010.

36. Kumar S, Stecher G and Tamura K: MEGA7: Molecular evolutionary genetics analysis version 7.0 for bigger datasets. Mol Biol Evol 33: 1870-1874, 2016.

37. Adzhubei IA, Schmidt S, Peshkin L, Ramensky VE, Gerasimova A, Bork P, Kondrashov AS and Sunyaev SR: A method and server for predicting damaging missense mutations. Nat Methods 7: 248-289, 2010.

38. Schwarz JM, Cooper DN, Schuelke M and Seelow D: MutationTaster2: Mutation prediction for the deep-sequencing age. Nat Methods 11: 361-362, 2014.

39. Kumar P, Henikoff S and Ng PC: Predicting the effects of coding non-synonymous variants on protein function using the SIFT algorithm. Nat Protoc 4: 1073-1081, 2009.

40. Blonska $\mathrm{M}$ and Lin $\mathrm{X}$ : NF- $\mathrm{\kappa B}$ signaling pathways regulated by CARMA family of scaffold proteins. Cell Res 21: 55-70, 2011.

41. Lenz G, Davis RE, Ngo VN, Lam L, George TC, Wright GW, Dave SS, Zhao H, Xu W, Rosenwald A, et al: Oncogenic CARD11 mutations in human diffuse large B cell lymphoma. Science 319: $1676-1679,2008$

42. Kan Z, Zheng H, Liu X, Li S, Barber TD, Gong Z, Gao H, Hao K, Willard MD, Xu J, et al: Whole-genome sequencing identifies recurrent mutations in hepatocellular carcinoma. Genome Res 23: 1422-1433, 2013

43. Arai E, Sakamoto H, Ichikawa H, Totsuka H, Chiku S, Gotoh M, Mori T, Nakatani T, Ohnami S, Nakagawa T, et al: Multilayer-omics analysis of renal cell carcinoma, including the whole exome, methylome and transcriptome. Int J Cancer 135: $1330-1342,2014$ 